\title{
Aplicação de Indicadores ROI e TCO (Custo Total) em Investimentos em ERP: Evidências em uma Instituição de Ensino Superior
}

Rosângela Venâncio Nunes
Mestrado em Logística e Pesquisa Operacional pela Universidade Federal do Ceará -
UFC Contadora, Consultora e Professora do Centro Universitário Estácio do Ceará e da Faculdade Ari de Sá - FAS Rua Sebastião de abreu, 177, apto 107, bloco C. Maraponga. Fortaleza/CE. CEP:

$60.710-830$

E-mail: angelnuness@gmail.com

André Felipe Pereira Alves Bacharelado em Ciências Contábeis pelo Centro Universitário Estácio do Ceará Rua Visconde de Mauá, 1940. Meireles. Fortaleza/CE. CEP: 60125-160 E-mail: andrefelipe0@me.com

Nayana de Almeida Adriano Doutorado em andamento em Administração e Controladoria pela Universidade Federal do Ceará - UFC Endereço Rua Gustavo Sampaio, 1413, apto. 1701. São Gerardo. Fortaleza/CE. CEP: 60.455-001 E-mail: nayanaadriano@hotmail.com

Charles Washington Costa de Assis Especialização em andamento em Contabilidade, Auditoria e Gestão Tributária pelo Instituto de Pós-Graduação - IPOG Profissão: Contador e Consultor na Acopeme Assessoria Contábil e Professor no

Centro Universitário Estácio do Ceará Rua Sebastião de abreu, 177, apto 107, bloco C. Maraponga. Fortaleza/CE. CEP: $60.710-830$ E-mail: charles-cont@hotmail.com

Rita de Cássia Fonseca Doutorado em andamento em Tecnologia e Sociedade pela Universidade tecnológica Federal do Paraná - UTFPR Professora da Universidade Estadual do Centro-Oeste - UNICENTRO Rua Tiradentes 1631. Bairro Centro. Guarapuava/PR. CEP: 85.010-30 E-mail: ritadecfonseca@gmail.com 
Aplicação de Indicadores ROI e TCO (Custo Total) em Investimentos em ERP: Evidências em uma Instituição de Ensino Superior Rosângela Venâncio Nunes, André Felipe Pereira Alves, Nayana de Almeida Adriano, Charles Washington Costa de Assis, Rita de Cássia Fonseca

\section{RESUMO}

O objetivo do trabalho é examinar se há benefícios no emprego de indicadores $\mathrm{ROI}$ e TCO para análise de viabilidade de investimento em ERP. Para isto, realizou-se estudo de caso em Instituição de Ensino Superior do Ceará com foco no retorno financeiro e no gerenciamento dos custos de implantação, sendo realizada verificação por meio de documentos da Universidade e de informações coletadas junto às áreas envolvidas. A investigação mostrou que o uso destes indicadores oferece vantagens, como por exemplo, visão mais clara do custo/benefício na adoção de novo ERP, e seus possíveis riscos, proporcionando maior segurança na decisão do investimento.

Palavras-chave: ERP. ROI. TCO. Análise de Investimento. IES.

\section{Application of ROI and TCO (Total Cost) Indicators in ERP Investiments: Evidence in Higher Education Institution}

\section{ABSTRACT}

The objetive of the work is to examine if there are benefits in the use of ROI and TCO indicators to analyze the viability of investment in ERP. To this end, a case study was carried out at a Higher Education Institution in Ceará with a focus on financial returns and on the management of implementation costs, with verification carried out through University documents and information collected from the areas involved. Research has shown that the use of these indicators offers advantages, such as, for example, a clearer view of the cost / benefit in adopting a new ERP, and its possible risks, providing greater security in the investment decision.

Keywords: ERP. ROI. TCO. IES.

\section{INTRODUÇÃO}

As pressões competitivas sofridas pelas empresas provocadas pelo processo de globalização resultaram na busca de alternativas para a redução de custos e diferenciação de produtos e serviços. Alternativas estas, que tornou necessária uma expressiva modificação da maneira como os setores das entidades interagiam, bem como da forma em que a informação seria gerada e disponibilizada para todos os seus 
Aplicação de Indicadores ROI e TCO (Custo Total) em Investimentos em ERP: Evidências em uma Instituição de Ensino Superior Rosângela Venâncio Nunes, André Felipe Pereira Alves, Nayana de Almeida Adriano, Charles Washington Costa de Assis, Rita de Cássia Fonseca

interessados. Tornando então imprescindível a utilização de ferramentas tecnológicas. De acordo com Zwicker e Sousa (2003), nos anos 90 assistiu-se ao surgimento e expressivo crescimento dos sistemas ERP no mercado de soluções de softwares empresariais.

Os sistemas ERP são conhecidos como sistemas de informação integrada ou Enterprise Resource Planning (ERP), que significa Planejamento de Recursos Empresariais. De acordo com Batista (2006), pode-se ter a seguinte definição para sistemas ERP: "Uma arquitetura de sistemas de informação que facilita o fluxo de informações entre todas as atividades da empresa."

Muitos autores como Chopra e Meindl (2003), Stair (2000), Laudon e Laudon (2007), O’Brien (2006) corroboram em suas pesquisas que o sistema ERP oferece uma forte integração de informação e simplifica os processos levando a empresa a ser eficiente e eficaz, unificando as relações entre os colaboradores e gestores, tornandose uma ferramenta essencial na sustentabilidade da mesma.

Contudo, Roseli e Cerqueira (2016), ao analisar o impacto do sistema ERP, em uma empresa do comércio exterior, concluem que os resultados de aprimoramento de processos e integração nos setores de importação e exportação com um sistema menos complexo. A integração dos processos em todas as unidades de negócios da empresa ainda não foi possível visualizar.

Nesse sentido, Padilha e Marins (2005), ressaltam que investimentos em sistemas ERP's possuem custos elevados, destacando-se os custos de hardware e infraestrutura computacional, de aquisição da licença de uso do ERP e também treinamento e consultoria para a implantação.

Assim sendo, as empresas de software e de consultorias, utilizam-se dessas ferramentas para avaliar os custos e o retorno que as empresas terão ao investir nos produtos oferecidos, a finalidade é mostrar o quanto esses instrumentos são importantes para auxiliar no processo de decisão e se serão viáveis frente ao retorno esperado. Dentre as ferramentas disponíveis para auxiliar estes estudos de viabilidade 
Aplicação de Indicadores ROI e TCO (Custo Total) em Investimentos em ERP: Evidências em uma Instituição de Ensino Superior Rosângela Venâncio Nunes, André Felipe Pereira Alves, Nayana de Almeida Adriano, Charles Washington Costa de Assis, Rita de Cássia Fonseca

têm-se Return on Investiment (ROI) ou Retorno sobre investimento e o Total Cost of Ownership (TCO) ou Custo Total de Propriedade, focos deste artigo.

Segundo concluíram Lessa, Clericuze e Lessa Filho (2007) a análise do TCO não oferece, em seu estágio atual, robustez para a avaliação completa das relações de custo-benefício em $\mathrm{TI}$, tomando como base somente a economia de custos e presumindo que os benefícios de todas as alternativas sejam semelhantes. Um exemplo disso é o caso do dimensionamento equivocado de configurações, quando da aquisição de software ou hardware, que pode levar ao acréscimo dos custos e não é contemplado, ainda, pelo TCO.

Longo e Sakata (2018), destaca a importância, no contexto atual, da análise do Custo Total de Propriedade, ou Total Cost Ownership (TCO), especialmente para a gestão de investimentos em Tecnologia de Informação (TI), pois auxilia para compreender melhor o ambiente empresarial.

Segundo Giacomazi (2004), o custo total de propriedade, é uma das ferramentas que ajudam a projetar uma estimativa dos custos diretos e indiretos, e também dos gastos com a manutenção que as empresas terão com a compra de novos investimentos em tecnologia da informação, mostrando-se assim uma ferramenta de extrema importância para auxiliar os administradores a buscarem novas alternativas, sem a necessidade de gastos dispensáveis e para que se possam manter competitivos no mercado.

O retorno sobre investimento é um tema com uma enorme relevância para as entidades nos dias de hoje, pois o mercado atual gira em torno de inovações e dos investimentos feitos pelas empresas, a fim de conseguir um retorno financeiro ou econômico sobre eles. Apesar dos softwares hoje serem investimentos comuns nas empresas, elas possuem um grande desafio que é atender seus fornecedores e clientes, com as melhores soluções, com o menor custo gerando os melhores resultados possíveis. 
Aplicação de Indicadores ROI e TCO (Custo Total) em Investimentos em ERP: Evidências em uma Instituição de Ensino Superior

Rosângela Venâncio Nunes, André Felipe Pereira Alves, Nayana de Almeida Adriano, Charles Washington Costa de Assis, Rita de Cássia Fonseca

Com o objetivo de aperfeiçoar essas informações, ou simplesmente para se ter uma maior visão das operações, as empresas tendem cada vez mais em investir em Tecnologia da Informação (TI). Junto com essa necessidade, e levando em consideração que as despesas operacionais com infraestrutura de TI vêm aumentando consideravelmente, é, portanto, necessário calcular a viabilidade econômica desses investimentos.

Estimar os custos de investimento em um Sistema ERP é relativamente simples, no entanto, calcular os benefícios deste investimento, como seu retorno tanto nas formas tangíveis e intangíveis, inclui uma série de fatores, como o tempo que leva para ocorrer, a adaptação da empresa, o custo da oportunidade, entre outros.

A partir do que foi contextualizado, este estudo pretende responder a seguinte indagação: quais são os benefícios da aplicação dos indicadores ROI e TCO como ferramentas de análise de viabilidade de investimentos em um sistema de informações como o ERP em uma Instituição de Ensino Superior (IES)?

Nesse sentido, o presente estudo tem como objetivo geral analisar se há benefícios da aplicação dos indicadores ROI e TCO para análise de viabilidade de investimentos em um sistema de informações como o ERP em uma instituição de ensino superior do Ceará.

\section{REFERENCIAL TEÓRICO}

Segundo Haberkorn (2003), o ERP possui, porém, mais funcionalidade e interatividade que o MRP e o MRP II, pois uma empresa não é constituída somente por máquinas e materiais, e sim, parte integrante e controlada por módulos financeiros, contábil e pessoas. Daí surgiu a necessidade de integrar todos os setores empresariais, alavancando o surgimento dos Sistemas Integrados de Gestão, onde foram acrescentados diversos módulos que permitem a administração não somente da manufatura, mas de toda a organização. O ERP é o nome dado a este tipo de solução, 
Aplicação de Indicadores ROI e TCO (Custo Total) em Investimentos em ERP: Evidências em uma Instituição de Ensino Superior

Rosângela Venâncio Nunes, André Felipe Pereira Alves, Nayana de Almeida Adriano, Charles Washington Costa de Assis, Rita de Cássia Fonseca

a integração e informatização de todos os processos da empresa sejam eles contábeis, financeiros, de $\mathrm{RH}$, estoques, custos etc.

Segundo Souza e Saccol (2003), Os ERP's são sistemas de informação adquiridos na forma de pacotes comercias de software que permitem a integração de dados dos sistemas de informação transacionais e dos processos de negócios ao longo de uma organização.

Os ERP's são sistemas de gestão empresarial, ou seja, um único sistema para auxiliar no processo de controle de uma entidade. O ERP possui vários subsistemas como, subsistema de contas a pagar, subsistema de contas a receber, subsistema de contabilidade, entre outros. Usando apenas um sistema que integra todos os setores a comunicação interna se torna mais fácil e o custo é reduzido. $O$ departamento financeiro, por exemplo, pode saber rapidamente quanto dinheiro precisa em caixa para o pagamento de impostos e quanto direcionar ao pagamento de funcionários, de acordo com as informações que o setor de gestão de pessoas disponibilizar no sistema.

O sistema Enterprise Resource Planning ou Planejamento dos Recursos Empresariais é um software aplicativo que permite a empresa automatizar e integrar seus processos de negócios em um único banco de dados. O ERP também possibilita compartilhar dados e uniformizar processos de negócios e produzir e utilizar informações em tempo real (Colangelo Filho, 2001).

A utilização de sistemas ERP otimiza o fluxo de informações e facilita o acesso aos dados, favorecendo a adoção de estruturas organizacionais mais enxutas e flexíveis. Além disso, as informações tornam-se mais consistentes, possibilitando a tomada de decisão com base em dados que refletem a realidade da empresa.

Dentre seus benefícios, pode-se citar as melhorias gerenciais, que facilitam no processo decisório dos administradores, maior segurança na informação, pois unifica em um sistema todas as informações dificultando a perda de informações e possíveis fraudes, redução nos custos, que auxilia na otimização de tarefas diárias reduzindo os 
Aplicação de Indicadores ROI e TCO (Custo Total) em Investimentos em ERP: Evidências em uma Instituição de Ensino Superior

Rosângela Venâncio Nunes, André Felipe Pereira Alves, Nayana de Almeida Adriano, Charles Washington Costa de Assis, Rita de Cássia Fonseca

custos operacionais, e o aumento da competitividade, pois com todas essas vantagens, a empresa fica mais confiante melhorando a qualidade do produto e consequentemente o seu poder de competição.

Mas o ERP também possui suas desvantagens, como a dependência dos módulos, pois um acaba dependendo do outro, gerando um maior controle sobre os usuários, aumentando à resistência a mudança podendo gerar desmotivação por parte dos funcionários demonstrou que, embora o sistema SAP seja considerado uma ferramenta muito importante para o gerenciamento, a sua implementação foi caracterizada por riscos associados à necessidade de obter reconhecimento institucional e aceitação interna

O trabalho de Santos e Caliman (2019) reforça esse entendimento, pois demonstrou que, embora o sistema SAP seja considerado uma ferramenta muito importante para o gerenciamento, a sua implementação foi caracterizada por riscos associados à necessidade de obter reconhecimento institucional e aceitação interna em uma empresa de logística e transporte.

Os sistemas são desenvolvidos a partir de modelos-padrões de processos, que não atendem a determinada necessidade, forçando a empresa a adequá-lo ao seu processo, gerando custos adicionais. Davenport (1998) afirma que é o fornecedor que define o que é melhor, e não o cliente. Assim, com a maior exigência do mercado é necessário que empresas proporcionem maiores recursos para seus clientes.

\subsection{Os custos de um Sistema ERP}

Wagle (1998) recomenda que a decisão de implantar o ERP só deve ser tomada com base em um fluxo de caixa positivo, pois se tratam de projetos nos quais o período de retorno do investimento (payback) é muito longo e o investimento muito grande.

No entanto, apesar dos benefícios aparentemente óbvios da TI, Basso, Maçada, Pinto e Luanardi (2020) salientam que há dúvidas sobre seu impacto, especialmente 
Aplicação de Indicadores ROI e TCO (Custo Total) em Investimentos em ERP: Evidências em uma Instituição de Ensino Superior Rosângela Venâncio Nunes, André Felipe Pereira Alves, Nayana de Almeida Adriano, Charles Washington Costa de Assis, Rita de Cássia Fonseca

nas pequenas e medias empresas como estudos de caso para recuperar os investimentos em sistemas tecnológicos

Padilha e Marins (2005) citam alguns custos de um ERP relativos à implantação e a sua manutenção, como Custo de Treinamento, Integração e testes, Conversão de dados e Horas de Consultoria.

Diante do cenário atual, as empresas se tornam reféns desses sistemas integrados, por isso é de grande valia que saibam quanto irão desembolsar na implantação de um ERP e também após essa integração, tentando estimar os custos que terão e em quanto tempo serão compensados com a decisão desse investimento, podendo-se utilizar de algumas ferramentas para essa análise.

\subsection{Investimentos em sistemas de informação: ferramentas para análise de viabilidade}

Ao considerar investimento é todo o capital desprendido no desejo de se obter lucro. Segundo Megliorini e Vallim (2009), análise de investimento é o modo de antecipar, por meio de estimativas os resultados oferecidos pelos projetos. Empregar um conjunto de técnicas que possibilitem comprovar os resultados de diferentes alternativas e auxiliar a tomada de decisões.

Assaf Neto (2009) complementa que os métodos quantitativos de análise econômica de investimentos podem ser classificados em dois grandes grupos: os que levam em conta o valor do dinheiro no tempo e os que consideram essa variação por meio do critério do fluxo de caixa descontado. Exceção é geralmente feita, no entanto, ao método do tempo de retorno do investimento (período de payback) o qual apesar de ser formalmente enquadrado no primeiro grupo, tem grande importância decisória e permite ainda seu cálculo em termos de valor atualizado.

Segundo Wantroba (2007), os investimentos em TI devem ser constantes, no entanto, é preciso que sejam avaliados com muito cuidado, esse tipo de investimento costuma ser alto, especialmente quando se trata de investimento em equipamentos, 
Aplicação de Indicadores ROI e TCO (Custo Total) em Investimentos em ERP: Evidências em uma Instituição de Ensino Superior

Rosângela Venâncio Nunes, André Felipe Pereira Alves, Nayana de Almeida Adriano, Charles Washington Costa de Assis, Rita de Cássia Fonseca

contribuindo para que o problema se torne mais complexo, pois a depreciação desses equipamentos acontece de maneira muito rápida. Esses investimentos em TI contribuem para a melhoria do desempenho das empresas, pois os computadores vêm conquistando cada vez mais espaço e se transformaram em ferramenta estratégica para expansão e gestão de negócios, dessa forma a TI deve ser vista como um meio de viabilizar os Projetos de SI, interferindo em toda a organização.

Ao tomar a decisão pela utilização de sistemas ERP, as empresas esperam obter: a integração, o incremento das possibilidades de controle sobre processos da empresa, a atualização tecnológica, a redução de custos da informática, o acesso a informações de qualidade em tempo real (Wantroba, 2007).

Para a avaliação do ROI, os custos e despesas envolvidas devem ser cuidadosamente avaliados, bem como as receitas que podem advir deste novo recurso (Giacomazi, 2004).

Logo, o ROI pode ser apurado pela seguinte equação (Assaf Neto, 2009):

$$
R O I=\frac{\text { Lucro Operacional }}{\text { Investimento }}
$$

Pinheiro (2005) diz que o conceito do ROI evoluiu internamente em muitas empresas que criaram categorias de acordo com o tipo de investimento em $\mathrm{Tl}$, os que reduzem custos, os que investem em segurança, aqueles que criam rendimentos e ainda os que simplificam os processos.

Segundo Ellram (2002), o TCO é um importante instrumento de gerenciamento de custos usado pelas organizações e é definido como uma abordagem para se entender e gerenciar os verdadeiros custos que envolvem um bem a ser negociado com fornecedor; ou a decisão sobre terceirização. Utiliza-se o TCO em 
Aplicação de Indicadores ROI e TCO (Custo Total) em Investimentos em ERP: Evidências em uma Instituição de Ensino Superior Rosângela Venâncio Nunes, André Felipe Pereira Alves, Nayana de Almeida Adriano, Charles Washington Costa de Assis, Rita de Cássia Fonseca

diferentes graus, cobrindo uma gama de situações relacionadas a compras estratégicas e táticas.

De acordo com Pinheiro (2005b), o simples funcionamento de um computador na rede de uma empresa apresenta diversos encargos financeiros associados, como o custo do hardware, do sistema operacional, das aplicações e da manutenção do conjunto em utilização. O total desses encargos é denominado de Custo Total da Propriedade (Total Costs of Ownership) ou TCO.

O conceito de TCO requer que as empresas considerem as atividades pelas quais elas são responsáveis e as causas dessas atividades que incorreram em custos. O objetivo é que a empresa possa identificar o verdadeiro custo de qualquer atividade, ao invés de simplesmente alocar os custos (Ellram \& Siferd, 1998).

Pode-se perceber que uma implementação de TCO bem realizada pode influenciar em todas as áreas de uma empresa, desde o nível mais operacional até o mais estratégico. 
Aplicação de Indicadores ROI e TCO (Custo Total) em Investimentos em ERP: Evidências em uma

Instituição de Ensino Superior

Rosângela Venâncio Nunes, André Felipe Pereira Alves, Nayana de Almeida Adriano, Charles Washington Costa de Assis, Rita de Cássia Fonseca

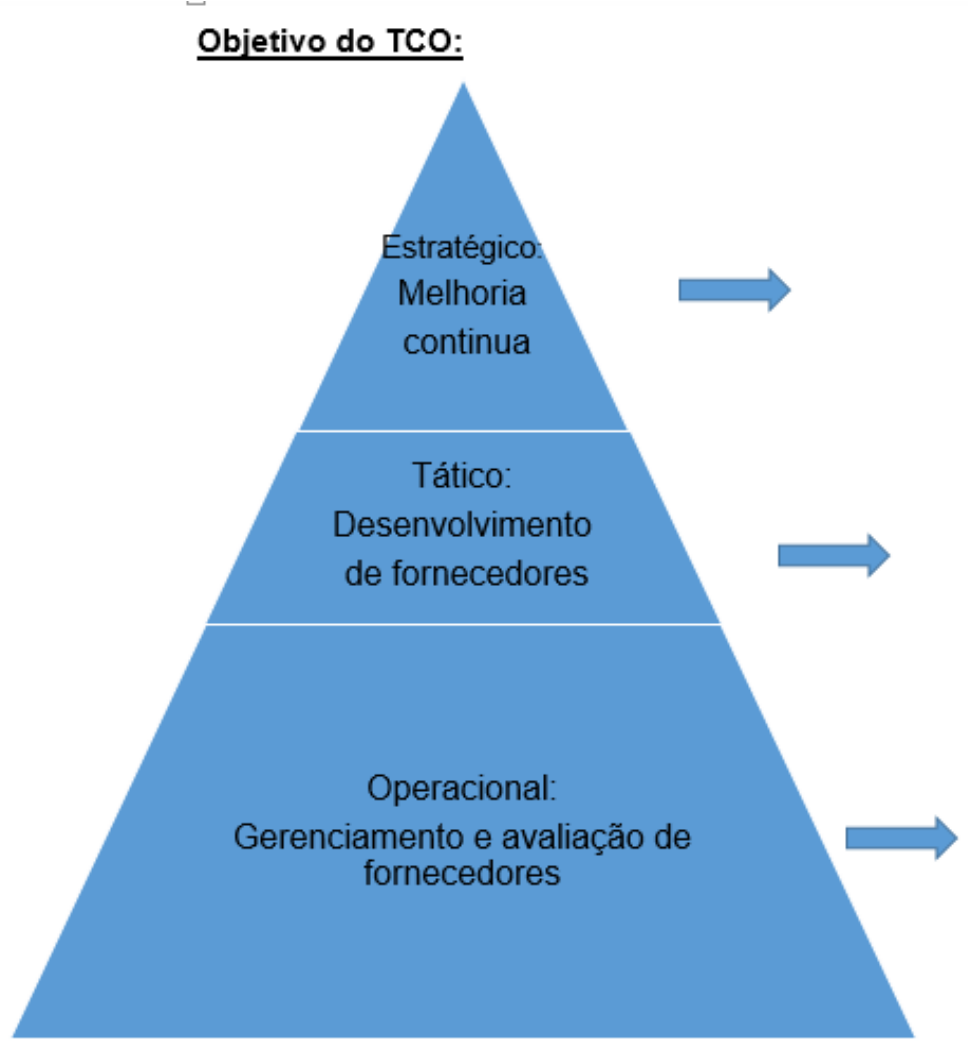

\section{Principais aplicações}

- Questionamento de processos fundamentais:

- Redefinição de processos;

- Decisões de outsourcing

- Gerenciamento de cadeias de suprimentos.

- Identificação de fatores que acarretam elevados custos tanto interna quanto externamente;

Feedback para fornecedores;

Acompanhamento de desempenho;

Seleção de fornecedores;

Alocação de volume de compras;

Alocação de custos a produtos;

Figura 1. Objetivos e áreas de influência do TCO.

Fonte: Santos (2011) adaptado pelos autores.

Para Santos Júnior e Gonçalves (2006), na prática para calcular TCO leva-se em conta o custo de aquisição dos equipamentos necessários, o treinamento dos funcionários, o custo/hora das manutenções e o custo de falhas. Caetano, Borinelli e Rocha (2019), reforçam a ideia da inclusão do custo de descarte como parte do custo total de propriedade.

TCO é realmente gestão estratégica de custos, quando ele ocorre no nível estratégico, como ajudar a melhorar os processos na organização ou na cadeia de informação. Assim, toda a análise de TCO é um suporte a gestão estratégica de custos. (Ellram \& Siferd, 1998).

Após a descrição das ferramentas utilizadas no estudo e com base nas citações acima o próximo passo é realizar a definição do objeto em estudo, dos procedimentos e 
Aplicação de Indicadores ROI e TCO (Custo Total) em Investimentos em ERP: Evidências em uma Instituição de Ensino Superior Rosângela Venâncio Nunes, André Felipe Pereira Alves, Nayana de Almeida Adriano, Charles Washington Costa de Assis, Rita de Cássia Fonseca

da coleta de dados que serão utilizados da empresa em questão, descrevendo da melhor forma como a investigação foi realizada e aplicando posteriormente no estudo de caso.

\section{PROCEDIMENTOS METODOLÓGICOS}

O objetivo dessa pesquisa é analisar os benefícios da aplicação das ferramentas ROI e TCO para o estudo de viabilidade na implantação de um sistema ERP em uma IES no Ceará.

Dando início a coleta de dados, a verificação foi realizada por meio de documentos da empresa e de informações adquiridas junto às áreas envolvidas, abordando a investigação dos critérios utilizados para a análise de investimentos, pois cada estudo de caso deve se basear em uma estratégia geral estabelecendo prioridades sobre o que será analisado e por quê (Yin, 2005).

Através de um estudo documental, foram coletadas informações oriundas dos orçamentos, dos balanços patrimoniais e das demonstrações de resultados do exercício da empresa dos anos de 2007 a 2012 para a análise dos custos e cálculos dos indicadores de viabilidade de investimentos, antes e após a implantação, tendo que vista que a mesma ocorreu no ano de 2011. Estas informações foram base para avaliação dos custos do sistema (desde a aquisição) e para elaboração de quadros de comparação de custos e a avaliação os aspectos financeiros (ROI) e outros índices matemáticos de retorno (como o TCO), conforme Figura 2. 
Aplicação de Indicadores ROI e TCO (Custo Total) em Investimentos em ERP: Evidências em uma Instituição de Ensino Superior Rosângela Venâncio Nunes, André Felipe Pereira Alves, Nayana de Almeida Adriano, Charles Washington Costa de Assis, Rita de Cássia Fonseca

\begin{tabular}{|c|}
\hline Software - Licença \\
\hline Software - Terceiros e Manutenção \\
\hline Customizações \\
\hline Hardware \\
\hline Desktops - Substituições \\
\hline Consultorias (Implantação) \\
\hline Equipes Implantação \\
\hline Mudança no perfil colaborador (Melhor qualificação) \\
\hline
\end{tabular}

Figura 2. Custos de implantação

Fonte: Autores baseado em Esteves et al. (2000).

Além dos custos facilmente visualizados, segundo Esteves et al. (2000), existem os custos intangíveis, que são denominados segundo a metodologia TCO, como ocultos. Esses custos estão relacionados às fases do ciclo de vida do sistema, logo também foram mapeados conforme a metodologia dos citados autores os custos tangíveis nas fases de adoção, aquisição, implementação, manutenção e evolução. Não se utilizou a fase de abandono, visto que o projeto não foi abandonado.

\section{ANÁLISE DE RESULTADOS}

\subsection{O Ambiente em Estudo}

A Instituição de ensino superior em questão iniciou suas atividades acadêmicas em agosto de 2001, com cursos de graduação nas áreas de Comunicação Social e Administração.

A instituição conta com várias práticas que incluem métodos modernos e inovadores baseados em workshops, debates, estudos de casos, fóruns e muitos outros. Tudo isso para que os alunos vivam as experiências necessárias para se tornarem profissionais prontos para ingressarem no mercado. 
Aplicação de Indicadores ROI e TCO (Custo Total) em Investimentos em ERP: Evidências em uma Instituição de Ensino Superior Rosângela Venâncio Nunes, André Felipe Pereira Alves, Nayana de Almeida Adriano, Charles Washington Costa de Assis, Rita de Cássia Fonseca

A instituição conta com 43 cursos de graduação e 08 de pós-graduação, possuindo duas filiais em Fortaleza.

O ERP utilizado pela instituição desde 2011 é o sistema SAP R/3. Este sistema é integrado e permite um planejamento e controle do negócio, visto que possui um número de atividades já moldadas e prontas para serem utilizadas. O SAP é um sistema de alta complexidade decorrente do fato de que ele considera como processo de negócio a totalidade da cadeia funcional envolvida no desenvolvimento do mesmo.

$\mathrm{Na}$ IES, as funções de trabalho estão divididas em áreas organizacionais e o ERP também está dividido desta forma, possuindo vários módulos. Os módulos são os menores conjuntos de funções que podem ser adquiridos e implementados separadamente no sistema. O sistema ERP da empresa é composto por um conjunto de módulos de software integrados interativamente. O sistema pode tratar atividades desde a cadeia de controle até relacionamento com os clientes da organização. $O$ sistema SAP na instituição é composto pelos seguintes módulos integrados:

- Módulo de finanças (FI) satisfaz todas as necessidades que deve cumprir o departamento de gestão financeira de uma empresa. O módulo $\mathrm{FI}$ apresenta ao colaborador os componentes que integram o livro razão, contas a pagar, contas a receber, contabilidade bancária, controle de ativos fixos, controle de orçamento etc;

- O módulo de Gestão de Materiais (MM), se integra completamente à outras áreas funcionais do SAP e dá suporte a todas as fases de planejamento das necessidades de consumo, planejamento e gestão de compras, gestão de serviços etc;

- O módulo de Controladoria (CO) proporciona informações para auxiliar nas decisões de gestão. Facilita a coordenação, o controle e a otimização de todos os processos em uma empresa. Entre os assuntos apresentados estão a contabilidade de classes de custo, de centros de custo, de custos por processos, 
Aplicação de Indicadores ROI e TCO (Custo Total) em Investimentos em ERP: Evidências em uma Instituição de Ensino Superior Rosângela Venâncio Nunes, André Felipe Pereira Alves, Nayana de Almeida Adriano, Charles Washington Costa de Assis, Rita de Cássia Fonseca

de centros de benefício, ordens $\mathrm{CO}$, controle de custo de produto, conta de resultados etc.

Tais módulos são divididos em subsistemas que executam uma ou mais tarefas e processos para um determinado departamento da empresa. Os programas realizam uma interação com os usuários, recebendo, processando e devolvendo os dados sobre fatos e eventos que ocorrem, como exemplo tem-se: movimentos de estoque, registro de transações financeiras, análise orçamentária, realização de pedidos de compras etc.

A Figura 3 expressa o um detalhamento do projeto do ERP na empresa.

\begin{tabular}{|l|l|}
\hline DETALHAMENTO DO PROJETO \\
\hline Nome do projeto & Projeto Join (Substituição do sistema atual por um ERP.) \\
\hline Descrição & $\begin{array}{l}\text { Projeto que visa à substituição dos sistemas atuais por outro capaz de } \\
\text { integrar toda a organização e facilitar o trabalho dos setores administrativos. } \\
\text { O Projeto deverá ser implantado em todas as unidades da organização e } \\
\text { abrangerá o, Ativo Fixo, Controladoria, Financeiro, Estoque e Compras. }\end{array}$ \\
\hline Impacto na organização & $\begin{array}{l}\text { Possível resistência às mudanças por parte dos usuários, risco de } \\
\text { descontinuidade do sistema e da não adaptabilidade, investimento alto. }\end{array}$ \\
\hline Usuários do Sistema & $\begin{array}{l}800 \text { usuários cadastrados; } \\
80 \text { usuários concorrentes; ou seja, somente } 80 \text { usuários conseguem acessar } \\
\text { o sistema ao mesmo tempo. }\end{array}$ \\
\hline
\end{tabular}

Figura 3. Detalhamento do projeto de implantação

Fonte: Dados da pesquisa, extraídos da empresa estudada.

O Projeto se trata de Substituição de Sistemas, pois o sistema que estava sendo utilizado encontrava-se bastante desgastado, causando manutenção excessiva o que depois de algum tempo, visto pelo lado econômico, seria necessário substituí-lo.

$\mathrm{Na}$ Figura são mostrados alguns riscos e vantagens do projeto, como uma possível resistência dos usuários, dificuldade de adaptação, etc. Mesmo assim, a diretoria resolveu investir em um novo sistema, pois além de todas as vantagens e recursos que um sistema de ponta oferece, ele ainda possui uma enorme quantidade 
Aplicação de Indicadores ROI e TCO (Custo Total) em Investimentos em ERP: Evidências em uma Instituição de Ensino Superior Rosângela Venâncio Nunes, André Felipe Pereira Alves, Nayana de Almeida Adriano, Charles Washington Costa de Assis, Rita de Cássia Fonseca

de licenças que no sistema anterior era limitado, e que agora irá oferecer maior número de acessos simultâneos, diminuindo o risco de uma queda na produtividade.

A seguir, na Tabela 1 tem-se um detalhamento de orçamento coma relação dos custos relacionados ao projeto, comparando o que se planejou e o que efetivamente foi realizado.

Tabela 1

Comparativo da Estimativa dos Custos de Implantação do Sistema ERP

\begin{tabular}{lcccc}
\hline \multicolumn{1}{c}{ Custos de Implantação } & $\begin{array}{c}\text { Gastos } \\
\text { Orçados } \\
\text { (31/12/2011 }\end{array}$ & $\begin{array}{c}\text { Gastos } \\
\text { Realizado } \\
\mathbf{3 1 / 1 2 / 2 0 1 1}\end{array}$ & $\begin{array}{c}\text { Variação } \\
\text { Orçado x } \\
\text { realizado }\end{array}$ & Variação \\
\hline Software - Licença & $295.000,00$ & $288.275,92$ & $6.724,08$ & 0,02 \\
Software - Terceiros e Manutenção & $39.440,00$ & $64.830,00$ & $-25.390,00$ & $-0,64$ \\
Customizações & $59.996,00$ & $92.155,72$ & $-32.159,72$ & $-0,54$ \\
Hardware & $112.000,00$ & $63.731,33$ & $48.268,67$ & 0,43 \\
Desktops - Substituições & $16.000,00$ & $9.026,22$ & $6.973,78$ & 0,44 \\
Consultoria (Implantação) & $224.800,00$ & $667.256,57$ & $-442.456,57$ & 1,97 \\
Equipes Implantação & $5.000,00$ & $4.534,33$ & 465,67 & 0,09 \\
Mudança no perfil colaborador & $88.000,00$ & $90.000,00$ & $-2.000,00$ & $-0,02$ \\
(Qualificação) & & & & $-\mathbf{0 , 5 2}$ \\
\hline Total Geral & $\mathbf{8 4 0 . 2 3 6 , 0 0}$ & $\mathbf{1 . 2 7 9 . 8 1 0 , 0 9}$ & $\mathbf{- 4 3 9 . 5 7 4 , 0 9}$ & \\
\hline
\end{tabular}

Nota. Fonte: Elaborado pelos autores com base nas informações cedidas pela empresa estudada

Com base nessas informações de avaliação dos custos do sistema (desde a aquisição), foram elaborados os quadros de comparação de custos e a avaliação os aspectos financeiros (ROI) e outros índices matemáticos de retorno (TCO, MVA), e feitas algumas considerações. Elaborou-se um gráfico, para avaliar as diferenças entre o previsto e o realizado (Figura 4).

A diferença no custo total é de $R \$ 439.574,09$, quase $52 \%$ acima do orçado. A diferença mais relevante que se verifica é referente à consultoria. Os usuários do 
Aplicação de Indicadores ROI e TCO (Custo Total) em Investimentos em ERP: Evidências em uma

Instituição de Ensino Superior

Rosângela Venâncio Nunes, André Felipe Pereira Alves, Nayana de Almeida Adriano, Charles Washington Costa de Assis, Rita de Cássia Fonseca

sistema não conseguiram avaliar previamente as horas que necessitariam, deixando esse item extrapolar, e muito, o que foi planejado. Talvez, o grande motivo tenha sido a dificuldade de adaptação do sistema para integrar a diversidade do grupo. Nos demais itens, observam-se uma discrepância menor, para mais ou para menos. Ressaltam-se alguns pontos que deixaram de ser observados devidamente pela organização:

- Os sistemas ERP são pacotes comerciais genéricos, desenvolvidos a partir de modelos padrões. Portanto, sua adaptação pode ou não ocorrer de forma satisfatória;

- A expectativa e a necessidade de mudança foram consideradas mais importantes que os custos que acarretariam, dessa forma, deixaram-se a desejar nas estimativas. Como os sistemas utilizados anteriormente apresentavam um custo alto de manutenção, procurou-se uma nova alternativa, "a qualquer custo", em busca da atualização tecnológica.

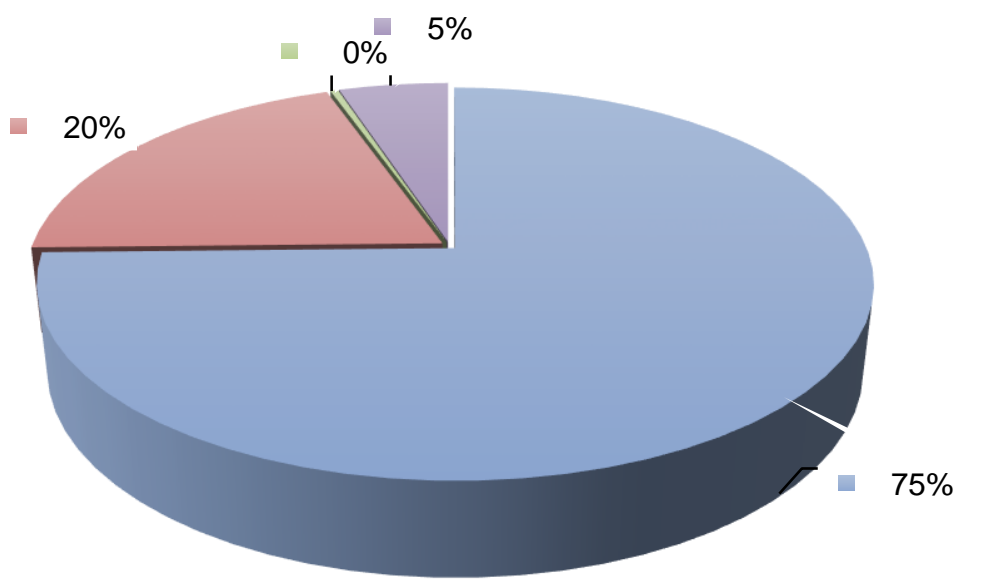

Aquisição

Evolução

- Implementação

Manutenção

Figura 4. Gráfico das Diferenças Previsto X Realizado

Fonte: Elaborado pelos autores com base nas informações cedidas pela empresa estudada 
Aplicação de Indicadores ROI e TCO (Custo Total) em Investimentos em ERP: Evidências em uma Instituição de Ensino Superior Rosângela Venâncio Nunes, André Felipe Pereira Alves, Nayana de Almeida Adriano, Charles Washington Costa de Assis, Rita de Cássia Fonseca

A fase de aquisição revela os maiores custos - com Consultoria e Licença $R \$$ 955.532,49, o que equivale a $75 \%$ dos custos totais apurados até o presente momento.

$\mathrm{Na}$ fase de Implementação - Equipes de implantação $\mathrm{R} \$ 4.534,33$ ou menos de $1 \%$ do total. Ressalte-se que nessa fase existem custos ocultos (não apurados adequadamente). Verificou-se também a existência de três keyusers, trabalhando em tempo integral na área de TI (o que não foi computado como custo de treinamento).

$\mathrm{Na}$ manutenção, os gastos previstos anualmente ficam em $\mathrm{R} \$ 64.830,00$.

$\mathrm{Na}$ fase de Evolução - estão previstos para o próximo ano, custos com customizações estimados em $\mathrm{R} \$ 92.155,72$, custos de melhoria em pessoal (melhor qualificação dos colaboradores) $\mathrm{R} \$ 90.000,00$ e substituição de desktops avaliados em 72.757,55 totalizando $\mathrm{R} \$ 254.913,27$. O volume e qualidade dos custos de implementação de sistemas ERP são relevantes na decisão sobre investimentos em TI. Devem ser analisados os aspectos referentes a toda vida útil do sistema, e não somente no período de aquisição.

\subsection{Aplicação das Ferramentas para a Análise da Viabilidade}

\subsubsection{Aplicação do ROI}

A IES em questão não utiliza as ferramentas do ROI e do TCO para o estudo de viabilidade de investimentos, mas o foco deste estudo é demonstrar que são peças importantes para o processo decisório de uma empresa.

Foi calculado com base nos dados extraídos da empresa o retorno sobre 0 investimento no decorrer de seis anos, como mostra a Tabela 2: 
Aplicação de Indicadores ROI e TCO (Custo Total) em Investimentos em ERP: Evidências em uma Instituição de Ensino Superior Rosângela Venâncio Nunes, André Felipe Pereira Alves, Nayana de Almeida Adriano, Charles Washington Costa de Assis, Rita de Cássia Fonseca

Tabela 2

Cálculo do ROI por período

\begin{tabular}{lcccccc}
\hline & $\mathbf{2 0 0 9}$ & $\mathbf{2 0 1 0}$ & $\mathbf{2 0 1 1}$ & $\mathbf{2 0 1 2}$ & $\mathbf{2 0 1 3}$ & $\mathbf{2 0 1 4}$ \\
\hline Lucro operacional & 1.447 & 1.512 & 1.587 & 1.756 & 1.819 & 1847 \\
Investimentos em T.I & 1.182 & 1.197 & 1.280 & 1.300 & 1.333 & 1354 \\
ROI & $22 \%$ & $26 \%$ & $24 \%$ & $35 \%$ & $36 \%$ & $36 \%$ \\
\hline
\end{tabular}

Nota. Fonte: Elaborado pelos autores com base nas informações extraídas da empresa.

Aplicando a fórmula do $\mathrm{ROI}$, dividindo o lucro operacional de cada ano pelo investimento realizado, verifica-se que no ano de 2012 foi gerado $35 \%$ de retorno, ano em que o novo sistema já estava em funcionamento na casa. Se compararmos2009 com 2014 (3 anos antes da implantação e 3 anos após) pode-se perceber que houve um aumento do $\mathrm{ROI}$ em $14 \%$, concluindo assim que o novo sistema proporciona de fato um maior retorno para a empresa. Como é visto, nos 3 anos anteriores a implantação o ROI é variável demonstrando que a empresa se mantém um pouco instável. Já após, é possível notar que ele teve um pequeno aumento de 2012 para 2013, e que depois se manteve constante, pois o retorno sobre o investimento em T.I é pequeno sendo analisado no curto prazo, mas no caso em especifico ainda é notável a melhora no resultado da empresa.

\subsection{Aplicação do TCO}

Fazendo a aplicação do TCO com base nos dados dos custos de implantação do ERP, é possível ver que os custos eram menores no sistema antigo (anterior a 2010), já os custos do sistema ERP na implantação são maiores em média 10\%, conforme mostrado na Tabela 3.

Fazendo o uso da ferramenta do TCO apenas pode-se mensurar os custos e proporcionar um maior controle deles, já que com essa ferramenta não se pode realizar um cálculo para estimar o retorno de investimento e nem o seu tempo. 
Aplicação de Indicadores ROI e TCO (Custo Total) em Investimentos em ERP: Evidências em uma Instituição de Ensino Superior

Rosângela Venâncio Nunes, André Felipe Pereira Alves, Nayana de Almeida Adriano, Charles Washington Costa de Assis, Rita de Cássia Fonseca

Comparando os dados dos custos na data base de 31/12/2010 e 31/12/2011(após implantação), nota-se que alguns deles não existiam, e com o novo sistema agora passam a aparecer nas demonstrações, confirmando que com o novo sistema vieram novos custos.

Tabela 3

Análise do TCO - Custos de implantação do ERP

\begin{tabular}{|c|c|c|c|c|}
\hline Custos & $\begin{array}{l}\text { Data base } \\
31 / 12 / 2010\end{array}$ & $\begin{array}{l}\text { Data base } \\
31 / 12 / 2011\end{array}$ & $\begin{array}{l}\text { Diferença } \\
\qquad \mathbf{R} \$\end{array}$ & $\%$ \\
\hline Software - Licença & $230.554,67$ & $288.275,92$ & $57.721,25$ & 0,25 \\
\hline Software - Terceiros e Manutenção & $86.768,89$ & $64.830,00$ & - $21.938,89$ & $-0,25$ \\
\hline Customizações & $56.686,85$ & $92.155,72$ & $35.468,87$ & 0,63 \\
\hline Hardware & $40.758,63$ & $63.731,33$ & $22.972,70$ & 0,56 \\
\hline Desktops - Substituições & - & $9.026,22$ & $9.026,22$ & 1,00 \\
\hline Consultorias (Implantação) & $736.867,32$ & $667.256,57$ & - $69.610,75$ & $-0,09$ \\
\hline Equipes Implantação & $10.785,56$ & $4.534,33$ & - $\quad 6.251,23$ & $-0,58$ \\
\hline $\begin{array}{l}\text { Mudança no perfil colaborador } \\
\text { (Melhor qualificação) }\end{array}$ & - & $90.000,00$ & $90.000,00$ & 1,00 \\
\hline Total Geral & $1.162 .421,92$ & $1.279 .810,09$ & $117.388,17$ & 0,10 \\
\hline
\end{tabular}

Nota. Fonte: Elaborado pelos autores com base nas informações extraídas da empresa em estudo

Para complementar que o investimento em um novo sistema foi realmente viável, com base em algumas contas do balancete referentes a T.I, é demonstrado que alguns custos diminuíram, mesmo outros tendo surgidos, o que mostra um sistema mais equilibrado e com baixo custo de manutenção. De acordo com a análise do balanço, nas formas horizontal e vertical demonstram-se os percentuais dos custos por conta, de acordo com Tabela 4.

Conforme mostra a análise horizontal (Tabela 4), os custos diminuíram significativamente, de um ano para o outro, demonstrando assim uma diminuição de $65 \%$ em relação ao total. 
Aplicação de Indicadores ROI e TCO (Custo Total) em Investimentos em ERP: Evidências em uma Instituição de Ensino Superior

Rosângela Venâncio Nunes, André Felipe Pereira Alves, Nayana de Almeida Adriano, Charles Washington Costa de Assis, Rita de Cássia Fonseca

Tabela 4

Análise horizontal dos custos com T.I

\begin{tabular}{|c|c|c|c|c|c|c|}
\hline & Análise horizon & do resultado do per & do (por & & & \\
\hline Conta & Descrição & $\begin{array}{l}\text { Saldo em 31.12.11 } \\
\text { (Sistema anterior) }\end{array}$ & $\mathrm{AH}(\%)$ & $\operatorname{AV}(\%)$ & $\begin{array}{c}\text { Saldo em } \\
31.12 .12 \\
\text { (SAP) }\end{array}$ & $\operatorname{AV}(\%)$ \\
\hline 421104 & Hora extra & $5.551,88$ & $-36 \%$ & $0 \%$ & $3.570,24$ & $0 \%$ \\
\hline 422201 & Consultorias & $431.812,87$ & $-61 \%$ & $23 \%$ & $167.229,32$ & $15 \%$ \\
\hline 422516 & $\begin{array}{l}\text { Manutenção em } \\
\text { sistemas }\end{array}$ & $68.070,88$ & $-43 \%$ & $2 \%$ & $38.711,93$ & $6 \%$ \\
\hline 421101 & $\begin{array}{l}\text { Salários e } \\
\text { ordenados }\end{array}$ & $1.408 .301,92$ & $-35 \%$ & & $910.058,00$ & \\
\hline 421117 & $\begin{array}{l}\text { Terceirização de } \\
\text { mão de obra }\end{array}$ & $5.683,17$ & $-100 \%$ & $75 \%$ & - & $79 \%$ \\
\hline \multirow[t]{2}{*}{ Total } & & $1.919 .420,72$ & $-42 \%$ & $0 \%$ & $1.119 .569,49$ & $0 \%$ \\
\hline & & & & $100 \%$ & & $100 \%$ \\
\hline
\end{tabular}

Nota. Fonte: Elaborado pelos autores com base nas informações extraídas da empresa em estudo

$\mathrm{Na}$ Tabela 4, observa-se que vários custos diminuíram após o primeiro ano de uso do novo sistema, como horas extras, consultorias, manutenção em sistemas e mais expressivamente nos salários dos colaboradores, pois mesmo a empresa tendo de contratar mão de obra mais qualificada a um custo mais elevado, foi visto que antes para desempenhar uma determinada atividade, era preciso vários funcionários, mas com a otimização do sistema esse número foi reduzido consideravelmente. Também foi reduzido o custo com a terceirização, pois antes o sistema não permitia que fossem feitas maiores analises de alguns processos, e a empresa era obrigada a contratar pessoal externo para realizá-las. 
Aplicação de Indicadores ROI e TCO (Custo Total) em Investimentos em ERP: Evidências em uma Instituição de Ensino Superior Rosângela Venâncio Nunes, André Felipe Pereira Alves, Nayana de Almeida Adriano, Charles Washington Costa de Assis, Rita de Cássia Fonseca

\section{CONSIDERAÇÕES FINAIS}

As formas usuais de avaliação de investimentos deixam a desejar quando o investimento se trata de um ERP. As fórmulas tradicionais de avaliação de investimentos não conseguem mensurar todos os benefícios de um sistema integrado como esse e por isso devem ser aliadas a outras técnicas. Esses benefícios são muito mais que somente financeiros, pois envolvem fatores estratégicos e intangíveis. Por esse motivo, as metodologias que consideram o valor intangível dos investimentos oferecem avaliações mais próximas à realidade.

Conforme a aplicação prática mostrou, os benefícios da utilização das ferramentas são de extrema importância para a empresa, pois complementam as técnicas já utilizadas mensurando com mais confiabilidade o retorno e o controle dos custos após a implantação de um novo sistema, como ressalta Basso et al. (2020), que descobriu que os investimentos em TI têm um impacto positivo no gerenciamento de clientes associado ao desempenho de custos, vendas e produtividade nas pequenas e médias empresas.

Respondendo ao problema da pesquisa, os resultados da pesquisa evidenciam alguns dos benefícios da usabilidade dessas ferramentas de sistema de informação, e a importância das mesmas, conforme sugere os resultados de Longo e Sakata (2018), pois foi possível identificar os investimentos efetuados em Sistemas de Informação, junto aos gestores das empresas frente a questionários da pesquisa do trabalho dos autores.

Esta pesquisa apresenta uma importante discussão sobre os investimentos em sistema de informação gerenciais, agregado a combinação de elementos estratégicos de análise de investimento com variáveis de desempenho.

Algumas restrições foram encontradas, no que diz respeito aos métodos de mensuração dos custos utilizados na empresa, fato que impossibilitou maiores análises sobre o assunto. Dentre as restrições pode-se citar o fato de que o sistema ERP vem 
Aplicação de Indicadores ROI e TCO (Custo Total) em Investimentos em ERP: Evidências em uma Instituição de Ensino Superior Rosângela Venâncio Nunes, André Felipe Pereira Alves, Nayana de Almeida Adriano, Charles Washington Costa de Assis, Rita de Cássia Fonseca

sendo utilizado pela instituição há pouco tempo (desde 2011) que fez com a análise ficasse limitada há um período curto de informação sobre geração de retornos.

Ressalta-se a dificuldade do retorno da implementação de um sistema de informação, como ERP, conforme descrito nos estudos de Giorgia e Santos (2015) que ao analisar à implantação, utilização e adaptação da ferramenta em uma empresa que já a utiliza os resultados evidenciam que o uso ainda mostra- que, a partir dos dados coletados foi possível identificar alguns fatores críticos que inibem a utilização e a adaptação do sistema como integração dos processos

Uma sugestão para futuras pesquisas seria a verificação de outras métricas ou até mesmo usar as mesmas, porém procurando um maior detalhamento desses instrumentos para a apuração de benefícios não identificadas nesse estudo, ressaltando, que há muito a ser explorado nesse assunto, até porque não existe ainda um consenso sobre ele.

\section{REFERÊNCIAS}

Assaf Neto, A. (2009) Finanças corporativas e valor. (4. ed.). São Paulo: Atlas.

Batista, E. O. (2006). Sistemas de informação: o uso consciente da tecnologia para o gerenciamento. São Paulo: Saraiva.

Basso, S. M., Maçada, A. C. G., Pinto, A. V., \& Lunsrdi, G. L. (2020). Impacto dos Investimentos em Tecnologia da Informação nas Variáveis Estratégicas Organizacionais e no Desempenho de Micro e Pequenas Empresas (MPEs). Revista de Empreendedorismo e Gestão de Pequenas Empresas, 9(2), 1-35.

Caetano, R. D., Borinelli, M. L., \& Rocha, W. (2019). Processo de aquisições na gestão pública brasileira: aplicação da metodologia de cálculo do custo total de propriedade. Anais do Congresso Brasileiro de Custos, Curitiba, Paraná, Brasil, 25.

Chopra, Sunil., \& Meindl, Peter. (2003) Gerenciamento da cadeia de suprimento: estratégia, planejamento e operação. São Paulo: Prentice Hall. 
Aplicação de Indicadores ROI e TCO (Custo Total) em Investimentos em ERP: Evidências em uma Instituição de Ensino Superior Rosângela Venâncio Nunes, André Felipe Pereira Alves, Nayana de Almeida Adriano, Charles Washington Costa de Assis, Rita de Cássia Fonseca

Colangelo Filho, L. (2001). Implantação de Sistemas ERP: um enfoque de longo prazo. São Paulo: Atlas.

Davenport, T. H. (1998, jul. ago). Putting de enterprise into the enterprise system. Harvard Business Review. 1221-1231. Recuperado de: <http://www.scielo.br/pdf/gp/v9n3/14570.pdf>. Acesso em: 23 out. 2015.

Ehrbar, A. (1999). EVA: Valor econômico agregado: a verdadeira chave para a criação da riqueza. Qualitymark Editora Ltda.

Ellram, L. M., \& Siferd, S. P. (1998). Total cost of ownership: a key conception in strategic cost management decisions. Journal of Bussines Logistics, 19 (1), 55-84. Recuperado de: <http://www.fatecsp.br/dti/tcc/tcc0041.pdf>. Acesso em: 25 out. 2015.

Ellram, L. M. (2002). Total Cost Modeling in Purchasing. CAPS. Recuperado de: <http://www.fatecsp.br/dti/tcc/tcc0041.pdf>. Acesso em: 25 out. 2015.

Esteves, J. M., Santos, A. A., \& Carvalho, J. A. (2000). O Ciclo de Vida dos Custos dos Sistemas ERP. Congresso Brasileiro de Custos. Recife, PE, Brasil, 7. Recuperado de:

$<\mathrm{http} / / /$ sol.ccsa.ufrn.br/ccsa/areas/cont/ppgcc/ftp_disciplinas_SIG/ERP_CicloVidaCu st os_VICBC.doc>. Acesso em: 25 out. 2015.

Giacomazi, L. C. (2004). O (ROI) Return on Investment sobre ERP: uma contribuição ao estudo da utilização do indicador como ferramenta para decisão de investimentos. Anais do Congresso Brasileiro de Custos, Porto Seguro, BA, 11. Recuperado de: <anaiscbc.emnuvens.com.br/anais/article/download/2295/2295>. Acesso em: 23 out. 2015.

Giorgia, M., \& Santos, W. S. (2015). Sistema ERP: um estudo exploratório numa empresa do setor têxtil do agreste pernambucano. Revista Brasileira de Gestão e Inovação, 2(3), 64-76.

Haberkorn, E. (2003). Gestão empresarial com ERP. São Paulo: Saraiva.

Laudon, K. C., \& Laudon, J. P. (2007). Sistemas de informações gerenciais. São Paulo: Pearson Prentice Hall.

Lessa, L. V. et al. (2007). Total Costs of Ownership (TCO): análise do custo total de propriedade em base comparativa entre os sistemas operacionais Windows $2000 \mathrm{e}$ Linux. Congresso Brasileiro de Custos, João Pessoa, PA, Brasil, 16. Recuperado 
Aplicação de Indicadores ROI e TCO (Custo Total) em Investimentos em ERP: Evidências em uma Instituição de Ensino Superior Rosângela Venâncio Nunes, André Felipe Pereira Alves, Nayana de Almeida Adriano, Charles Washington Costa de Assis, Rita de Cássia Fonseca

de: <http://anaiscbc.emnuvens.com.br/anais/article/viewFile/1447/1447>. Acesso em: 10 out. 2015.

Longo, L., \& Sakata, N. A. B. (2018). Custo Total de Propriedade (Total Cost Ownership) para Decisões de Investimentos em Tecnologia de Informação (TI). Revista Capital Científico - Eletrônica, 16(1), 20-35.

Megliorini, E., \& Vallim, M. A. (2009). Administração financeira: uma abordagem brasileira. São Paulo: Pearson.

O'brien, J. A. (2006). Sistemas de informação: e as decisões gerenciais na era da internet. (2a ed.). São Paulo: Saraiva.

Padilha, T. C. C., \& Marins, F. A. S (2005) Sistemas ERP: características, custos e tendências. Encontro Nacional de Engenharia de Produção, Curitiba, PR, Brasil, 22. Recuperado de: <http://www.scielo.br/pdf/prod/v15n1/n1a08.pdf>. Acesso em: 25 out. 2015.

Pinheiro, J. M. S. (2005). O retorno do investimento em projetos de redes. Recuperado de: <http://www.projetoderedes.com.br/artigos/artigo_roi.php>. Acesso em: 13 out. 2015.

Pinheiro, J. M. S. (2005b). A tecnologia da Informação e o custo total de propriedade. Recuperado de: <http://www.projetoderedes.com.br/artigos/artigo_tecnologia_da_informacao_e_o_c usto_total_de_propriedade.php>. Acesso em: 13 out. 2015.

Roselli, K., \& Cerqueira, L. S. (2016). Avaliação do Impacto da Implementação do Sistema ERP em uma Empresa de Comércio Exterior na Cidade de Rio Grande-RS. Revista de Tecnologia Aplicada, 5(3), 27-45.

Santos, I. C. V. (2011). Estudos de caso de custo total de propriedade TCO (Total cost of Ownership). Recuperado de: <http://www.fatecsp.br/dti/tcc/tcc0041.pdf> Acesso em: 25 out. 2015.

Santos, T. P., \& Caliman, D. R. (2019). Desafios da Implementação do Sistema de Controle e Gestão SAP em uma Empresa de Logística e Transporte. Gestão \& Conexões, 8(3), 90-108.

Santos Júnior, C. D. D., \& Gonçalves, M. A. (2006). Análise da substituição de um software proprietário por um software livre sob a ótica do custo total de propriedade: estudo de caso do setor de peças automobilísticas. Revista Contemporânea de Contabilidade, 3(6), 39-60 
Aplicação de Indicadores ROI e TCO (Custo Total) em Investimentos em ERP: Evidências em uma Instituição de Ensino Superior Rosângela Venâncio Nunes, André Felipe Pereira Alves, Nayana de Almeida Adriano, Charles Washington Costa de Assis, Rita de Cássia Fonseca

Souza, C. A., \& Saccol, A. Z. (2003) Sistemas ERP no Brasil (Enterprise Resource Planning): teoria e casos. São Paulo: Atlas.

Stair, R. M. (2000). Princípios de sistemas de informação: uma abordagem gerencial. (4a ed.). Rio de Janeiro: LTC.

Wagle, D. (1998) The case for ERP systems. The Mckinsey Quarterly, 2, 130-138 Recuperado de: http://www.abepro.org.br/biblioteca/ENEGEP2001_TR93_0166.pdf. Acesso em: 10 out 2015.

Wantroba, E. (2007). Avaliação de investimentos em sistemas integrados de gestão empresarial. Recuperado de: http:/http://www.pg.utfpr.edu.br/dirppg/ppgep/dissertacoes/arquivos/63/Dissertacao. pdf. Acesso em: 23 out. 2015.

Yin, R. K. (2005) Estudo de caso: planejamento e métodos. (2a ed.). Porto Alegre: Bookman.

Zwicher, R., \& Souza, C. A. (2003) Sistemas ERP: estudos de casos múltiplos em empresas brasileiras. In: Souza, C. A., \& Saccol, A. Z. (Org.). Sistemas ERP no Brasil: teoria e casos. São Paulo: Atlas. Recuperado de: <http://www.anpad.org.br/diversos/trabalhos/3Es/3es_2005/2005_3ES465.PDF>. Acesso em: 10 out. 2015.

Data de Submissão: 23/07/2018

Data de Aceite: 23/04/2020 